\title{
Effects of Early IL-17A Neutralization on Disease Induction in a Primate Model of Experimental Autoimmune Encephalomyelitis
}

\author{
Yolanda S. Kap • S. Anwar Jagessar • Nikki van Driel • \\ Erwin Blezer • Jan Bauer • Marjan van Meurs • \\ Paul Smith • Jon D. Laman • Bert A. 't Hart
}

Received: 30 June 2010 / Accepted: 28 July 2010 /Published online: 12 August 2010

(C) The Author(s) 2010. This article is published with open access at Springerlink.com

\begin{abstract}
We report on the effect of antibody-mediated neutralization of interleukin (IL)-17A in a non-human primate experimental autoimmune encephalomyelitis (EAE) model induced with recombinant human myelin oligodendrocyte glycoprotein (rhMOG). We tested a human-anti-human IL-17A-antibody in two doses (3 and $30 \mathrm{mg} / \mathrm{kg}$ ) against placebo (PBS). The treatment was started 1 day before EAE induction and continued throughout the experiment. Although all monkeys developed clinically evident EAE, the onset of neurological signs was delayed in some monkeys from both treatment groups. Total CNS lesion volumes, demyelination, or inflammation did not
\end{abstract}

Guarantor of the work: Dr. Bert A. 't Hart (Chairman of the Department of Immunobiology, BPRC)

Y. S. Kap · S. A. Jagessar · N. van Driel • P. Smith •

B. A. 't Hart $(\bowtie)$

Department of Immunobiology,

Biomedical Primate Research Centre,

P.O. Box 3306, 2280 GH Rijswijk, The Netherlands

e-mail: hart@bprc.nl

Y. S. Kap · M. van Meurs · J. D. Laman - B. A. 't Hart Department of Immunology, Erasmus Medical Centre, Rotterdam, The Netherlands

Y. S. Kap · S. A. Jagessar · M. van Meurs · J. D. Laman •

B. A. 't Hart

MS Centre ErasMS,

Rotterdam, The Netherlands

E. Blezer

Image Sciences Institute, University Medical Center Utrecht, Utrecht, The Netherlands

J. Bauer

Brain Research Institute, University of Vienna,

Vienna, Austria differ between the different groups. Immune profiling revealed an altered distribution of IL-17A producing cells in the lymphoid organs of antibody-treated monkeys. Comparable numbers of IL-17A producing cells were observed in the brain. RhMOG-induced $\mathrm{T}$ cell proliferation in the lymph nodes was slightly reduced after anti-IL-17A antibody treatment. To summarize, we found that anti-IL-17A antibody as a single treatment from disease induction effects a trend towards delayed neurological disease progression in the marmoset EAE model, although the effect did not reach statistical significance. This suggests a role of IL-17A in late stage disease in the marmoset EAE model, but IL-17A may not be the dominant pathogenic cytokine.

Keywords Th17 IL-23 - Antibody therapy ·

Non-human primate $\cdot$ Neuroimmunology

\section{Introduction}

Multiple sclerosis (MS) is a progressive neurological disease of unknown origin characterized by the progressive accumulation of inflammation and degeneration in the grey and white matter of the central nervous system (CNS). The accumulation of injury involves a combined cellular and humoral autoimmune attack on the protective myelin sheaths around axons leading to disturbed saltatory pulse conduction (Compston and Coles 2008; Sospedra and Martin 2005). This immunopathogenic process is modeled in the experimental autoimmune encephalomyelitis (EAE) model, an experimental disease induced in genetically susceptible laboratory animals including mice, rats, guinea pigs, and monkeys. The EAE model in common marmosets, a small-sized neotropical primate, combines a remarkable 
clinical and neuropathological similarity with MS and a high genetic and immunological proximity to humans ('t Hart and Massacesi 2009). The model is therefore exquisitely suitable for translational research into immunopathogenic mechanisms and the efficacy evaluation of therapeutic antibodies, which because of their high target specificity do not crossreact with lower species. The marmoset EAE model thus forms a bridge narrowing the gap between EAE models and MS ('t Hart et al. 2004; Kap et al. 2010).

Research conducted in the past years has shown that the $100 \%$ disease incidence and variable progression rate of neurological deficit is associated with the mobilization of highly reactive $\mathrm{T}$ cells present in the normal repertoire with variable specificities for myelin oligodendrocyte glycoprotein (MOG) (Jagessar et al. 2010; Kap et al. 2008). Whereas disease induction could be mapped to the CajaDRB1*W1201 restricted activation of T helper (Th)1 cells specific for $\mathrm{MOG}_{24-36}$ (Brok et al. 2000), the rate of disease progression appeared to be regulated by natural killer-like $\mathrm{T}$ cells specific for $\mathrm{MOG}_{34-56}$ (Jagessar et al. 2010; Kap et al. 2008). Further research confirmed that this $T$ cell subset is capable to induce clinical signs and pathology in the CNS white and grey matter without the support of innate antigen receptor ligands in the inoculum or autoantibodies against rhMOG or the inducing peptides (Jagessar et al. 2010). These data led to the hypothesis that $\mathrm{MOG}_{34-56}$-specific $\mathrm{T}$ cells have a central pathogenic role in the regulation of disease progression in the marmoset EAE model.

The most obvious immunological characteristics of $\mathrm{MOG}_{34-56}$-specific $\mathrm{T}$ cell lines generated from EAEaffected monkeys were specific cytolytic activity towards $\mathrm{MOG}_{34-56}$-pulsed target cells and production of IL-17A (Jagessar et al. 2010; Kap et al. 2008). However, typical Th1 cell-derived cytokines, such as interferon (IFN)- $\gamma$ or tumor necrosis factor alpha (TNF- $\alpha$ ), that are abundantly produced by the $\mathrm{MOG}_{24-36}$-specific Th1 cells involved in EAE initiation (Brok et al. 2000) were not produced by $\mathrm{MOG}_{34-56}$-specific $\mathrm{T}$ cells (Jagessar et al. 2010). This may well be due to the fact that for the activation of encephalitogenic Th1 cells in mice as well as in marmosets strong bacterial adjuvants are needed, i.e. complete Freund's adjuvant (CFA) (Billiau and Matthys 2001). By contrast, our data in the marmoset EAE model demonstrates that encephalitogenic $\mathrm{T}$ cells specific for $\mathrm{MOG}_{34-56}$ can also be activated by immunization with $\mathrm{MOG}_{34-56}$ in incomplete Freund's adjuvant (IFA) (Jagessar et al. 2010).

These observations prompted us to test whether antibody-mediated neutralization of IL-17A modulates disease progression in the EAE model in marmosets, elicited by a single immunization with recombinant protein of the extracellular domain of human MOG $\left(\mathrm{MOG}_{1-125}\right)$ in CFA. The most prevalent disease course in this model is clinically characterized by the sudden appearance of progressively accumulating motor defects after an episode of variable length, during which MRI-detectable lesions do not lead to evident neurological symptoms (Brok et al. 2000; Kap et al. 2008). A typical relapsing-remitting disease course is only occasionally observed. Our previous studies have revealed that intervention with an antibody against the shared p40 subunit of IL- 12 and IL- 23 has a clear beneficial effect in the rhMOG-induced EAE model ('t Hart et al. 2005b; Brok et al. 2002). This supports an important pathogenic role of IL-12 and IL-23, which may be exerted via the inhibition of IL-17A production, as IL-23 promotes IL-17A production (Langrish et al. 2005).

Here, we report that prophylactic treatment with anti-IL-17A monoclonal antibody $(\mathrm{mAb})$ at doses achieving plasma through levels above $1 \mu \mathrm{g} / \mathrm{ml}$ induced a moderate delay of EAE onset. These data indicate that IL-17A is only one of several cytokines driving progression of the marmoset EAE model to clinically evident neuroinflammatory disease; absence of IL-17A being possibly compensated by Th1 cytokines that are abundantly produced in the rhMOG/CFA model

\section{Material and methods}

\section{Animals}

The common marmoset monkeys used in this study were randomly selected from the outbred colony kept at the Biomedical Primate Research Centre (Rijswijk, The Netherlands) under conventional and not SPF conditions. Animals were only included after a complete physical, hematological, and biochemical check-up had been performed. During the study, monkeys were pair-housed in spacious cages and remained under intensive veterinary care. The daily diet consisted of commercial food pellets for New World monkeys (Special Diet Services, Witham, Essex, UK), supplemented with rice, raisins, peanuts, marshmallows, biscuits, fresh fruit, grasshoppers, and maggots. Drinking water was provided ad libitum. In accordance with the Dutch law on animal experimentation, all study protocols and experimental procedures have been reviewed and approved by the Institute's Ethics Committee.

The study comprised 22 male and two female monkeys, M05011 and M04049. The average age of the monkeys was $33 \pm 16$ months, which is adult age for marmosets. The average body weight was $377 \pm 35 \mathrm{~g}$.

\section{RhMOG-induced EAE}

EAE was induced by immunization with a recombinant protein encompassing the extracellular domain of human MOG residues 1-125, which was produced in Escherichia coli and purified as previously described (Kerlero de Rosbo 
et al. 1997). The inoculum, containing $100 \mu \mathrm{g}$ rhMOG in $300 \mu \mathrm{l}$ phosphate buffered saline (PBS) emulsified with $300 \mu \mathrm{l}$ CFA containing mycobacterium butyricum (Difco Laboratories, Detroit, MI), was injected at four locations into the dorsal skin under ketamin anesthesia $(40 \mathrm{mg} / \mathrm{kg}$; AST Pharma, Oudewater, The Netherlands).

Clinical signs were scored daily by two independent observers using a previously documented semiquantitative scale ('t Hart et al. 2008): $0=$ no evident clinical signs; $0.5=$ apathy, loss of appetite, altered walking pattern without ataxia; $1=$ lethargy, anorexia, tail paralysis, tremor; $2=$ ataxia, optic disease; $2.25=$ monoparesis; $2.5=$ paraparesis, sensory loss; $3=$ para- or hemiplegia. For ethical reasons monkeys were sacrificed before or once complete paralysis of hind limbs (score 23.0) was observed, or at the pre-determined endpoint of the study (post-sensitization day (psd) 113). Body weight measurements of conscious monkeys, which is used a surrogate disease marker, were performed three times per week.

Monkeys selected for necropsy were first deeply sedated by intramuscular injection of ketamin $(50 \mathrm{mg} / \mathrm{kg})$ and subsequently euthanised by infusion of pento-barbital sodium (Euthesate ${ }^{\circledR}$; Apharmo, Duiven, The Netherlands).

\section{Reactivity and dosing regimen of anti-IL-17A mAb}

The test substance was produced by UCB Celltech (UK) as a humanized IgG4 $\mathrm{K}$ mAb specific for human IL-17A, coded as 497.g2. The antibody has been extensively characterized in vitro in terms of bioassay, affinity for IL-17A, and cross-reactivity against marmoset IL-17A. The affinity of the antibody with marmoset IL-17A is twofold lower than with human IL-17A when assessed by Biacore and fourfold less potent in a bioassay compared with humans.

The animals were subcutaneously injected once a week starting 1 day before immunization until the predetermined end of the study at day 113. Animals were randomly assigned to three experimental groups. Eight animals received $3 \mathrm{mg} / \mathrm{ml} / \mathrm{kg}$ anti-IL-17A mAb diluted in PBS, eight animals were injected with $30 \mathrm{mg} / \mathrm{ml} / \mathrm{kg}$ antiIL-17A mAb diluted in PBS, and eight control animals received sterile PBS $(1 \mathrm{ml} / \mathrm{kg})$ as placebo treatment. All animals received the same volume per $\mathrm{kg}$ body weight. One monkey (M04063) in the $30 \mathrm{mg} / \mathrm{kg}$ antibody dose group succumbed at psd 69 unexpectedly without prior signs of EAE and was therefore excluded from further analyses. Autopsy revealed that the cause of death was not related to the test substance or EAE, but to perforation of the gastro-intestinal tract by plant material, possibly originating from the branches used for cage enrichment.
Blood sampling and plasma levels of anti-IL-17A mAb

Venous blood was collected into heparinized vacutainers (Greiner, Sölingen, Germany) under ketamin anesthesia (40 mg/kg; AST Pharma, Oudewater, The Netherlands) at psd $0,6,34,62$, and at necropsy.

After centrifugation plasma was collected and stored frozen at $-20^{\circ} \mathrm{C}$ until analysis of test substance levels was performed. Test substance plasma levels were determined by ELISA. Microtitre plates were coated with human IL17A (R\&D Systems, Minneapolis, MN) at $0.5 \mu \mathrm{g} / \mathrm{mL}$ in PBS overnight, blocked with PBS/1\% BSA, glazed with $\mathrm{PBS} / 5 \%$ lactose $/ 0.1 \%$ BSA, dried, sealed in foil pouches, and stored at $2-8^{\circ} \mathrm{C}$. The standard curve was prepared by making serial doubling dilutions of the 497.g2 top standard (starting at $200 \mathrm{ng} / \mathrm{mL}$ ) in $\mathrm{PBS} / 1 \% \mathrm{BSA} / 1 \% \mathrm{BGG} / 1 \%$ human plasma. $50 \mu \mathrm{L}$ of each standard, interassay control (IAC), and sample (diluted at least $1 / 100$ ) were added to the appropriate wells containing $50 \mu \mathrm{L}$ PBS/ $1 \%$ BSA $/ 1 \%$ BGG. The IAC concentrations were nominally 80,20 , and $8 \mathrm{ng} / \mathrm{mL}$. Standards, IAC, and samples were tested in duplicate. The plate was covered and incubated with agitation at RT for $2 \mathrm{~h}$. The plate was washed with PBS/0.1\% Tween- 20 four times and incubated with goat anti-human Kappa-HRP conjugate $(1 / 10,000)$ in $\mathrm{PBS} / 1 \% \mathrm{BSA} / 1 \%$ BGG at RT for $30 \mathrm{~min}$. The plate was washed again with PBS/ $0.1 \%$ Tween20 four times and incubated with $100 \mu \mathrm{L}$ Tetramethyl benzidine substrate for $10 \mathrm{~min}$. The reaction was stopped with $50 \mu \mathrm{L} /$ well of $2.5 \mathrm{M} \mathrm{H}_{2} \mathrm{SO}_{4}$ and measured at $450 \mathrm{~nm}$ (and $630 \mathrm{~nm}$ as a reference).

\section{Magnetic resonance images}

Post-mortem magnetic resonance images (MRI) of one brain hemisphere were recorded to assess differences in the CNS lesion load between treated and control monkeys (Blezer et al. 2007). Half of the brain collected at necropsy was fixed in $4 \%$ buffered formalin and transferred into buffered saline containing sodium azide after two weeks.

MRI experiments were performed on a $9.4 \mathrm{~T}$ horizontal bore MRI scanner (Varian, Palo Alto, CA). The formalinfixed brains were submerged in a non-magnetic oil (Fomblin; perfluorinated polyether, Solvay Solexis, Weesp, The Netherlands) to prevent unwanted susceptibility artifacts.

The following quantitative images were obtained (field of view $=2.5 \times 2.5 \mathrm{~cm}^{2}$, matrix $=256 \times 256$, slice thickness $=$ $0.75 \mathrm{~mm}$, number of slices $=41$, number of experiments $=2$ );

- $\mathrm{T}_{2}$ maps, which were calculated from the images obtained of a multi echo sequence using the following parameters: repetition time $=4,000 \mathrm{~ms}$, echo spacing $=$ 
$14.75 \mathrm{~ms}$, echo train length $=4 . \mathrm{T}_{2}$ maps were the result of a mono-exponential fit of the MRI signal intensities as a function of TE. The $\mathrm{T}_{2}$-weighted images with TE of $14.75 \mathrm{~ms}$ were used for region of interest (ROI) analyses.

- Magnetization transfer ratio (MTR) maps were calculated from two T1-weighted spin echo images with and without a magnetization transfer-saturation pulse, with $\mathrm{MTR}=100 *[$ (Munsaturated-Msaturated)/Munsaturated]. Repetition time $=1675 \mathrm{~ms}$; echo time $=$ $23 \mathrm{~ms}$; MT-pulse: $8.19 \mathrm{~ms}$ Gaussian-shaped pulse, nominal flip angle 1,000 , offset $-9.4 \mathrm{kHz}$.

ROI were defined using the free available medical image processing, analysis and visualization (MIPAV version 4.3.0, National Institutes of Health, Bethesda, MD) package. ROI of lesions, defined as areas with abnormal increased signal intensities, were automatically outlined in all T2-weighted images containing white matter structures using the level-set method of MIPAV. Volumes, T2, and MTR values were calculated from these ROI.

Histology and immunohistochemistry

Frozen and fixed tissues were processed for histological and immunohistochemical techniques as previously described ('t Hart et al. 1998; Laman et al. 1998).

Demyelination was visualized by staining myelin and myelin degradation products with Klüver Barrera method (Luxol Fast Blue with Periodic Acid-Schiff). Inflammation was visualized by staining infiltrating cells with hematoxylin and eosin. Of each animal eight sections, approximately $6 \mathrm{~cm}^{2}$ in total, were analyzed.

Snap-frozen sections of the brain, spleen, and axillary (ALN), inguinal (ILN), and cervical lymph nodes (CLN) were used to determine the number of IL-17A-producing cells. Sections of $6 \mu \mathrm{m}$ were thaw mounted on gelatin/ chrome alum coated glass slides and stored overnight in humidified atmosphere. Next day, sections were air-dried for $1 \mathrm{~h}$ at room temperature. Within 2 weeks sections were fixed at room temperature in fresh acetone containing $0.02 \% \mathrm{H}_{2} \mathrm{O}_{2}$. After air-drying, sections were washed with PBS containing $0.05 \%$ Tween-20 (Sigma-Aldrich Chemie, Zwijndrecht, The Netherlands) and incubated with 1\% blocking reagent (TSA HRP kit, Invitrogen, Molecular Probes, Carlsbad, CA) at room temperature for $1 \mathrm{~h}$. After blocking, sections were incubated for $1 \mathrm{~h}$ with a primary antibody against IL-17A (eBio64Cap17, eBiosciences, San Diego, CA). All incubations were performed at room temperature and between incubations sections were washed with $1 \%$ blocking reagent. Primary antibody was detected by incubation for $1 \mathrm{~h}$ with biotinylated rabbit anti-mouse IgG (DAKO, Glostrup, Denmark) and streptavidin-HRP (TSA HRP kit, Invitrogen) for $30 \mathrm{~min}$, both diluted in $1 \%$ blocking reagent. Sections were incubated for $10 \mathrm{~min}$ with tyramide-biotin dissolved in amplification buffer (TSA HRP kit, Invitrogen) containing $0.15 \% \mathrm{H}_{2} \mathrm{O}_{2}$. Next, sections were again incubated for $30 \mathrm{~min}$ with streptavidin-HRP. To reveal peroxidase activity, sections were incubated with AEC (3-amino-9-ethylcarbazole, Sigma) substrate for 10 min resulting in a bright red precipitate. Sections were counterstained with hematoxylin and embedded with glycerol-gelatin. In control stainings in which the first antibody was omitted or using an isotype control, no immunopositive cells could be detected. For the lymph nodes, the number of IL-17A producing cells is the mean of the number of IL-17A-positive cells of three nonadjacent sections.

Analysis of T cell reactivities

Ex vivo $\mathrm{T}$ cell responses against different antigens were analyzed as described previously (Brok et al. 2000). Briefly, PBMC were isolated by density gradient centrifugation over lymphocyte separation medium (AxisShield PoC AS, Oslo, Norway). Mononuclear cell (MNC) suspensions were prepared from aseptically removed spleen and ALN. Cells were cultured in triplicate for detection of proliferative responses towards rhMOG and a panel of MOG peptides. All antigens were tested at $5 \mu \mathrm{g} / \mathrm{ml}$. After $48 \mathrm{~h}$ of culture, $0.5 \mu \mathrm{Ci} /$ well of tritiated thymidine $\left({ }^{3} \mathrm{H}\right.$-Thy) was added and incorporation of radiolabel was determined after $18 \mathrm{~h}$ using a matrix $9600 \beta$-counter (Packard 9600; Packard Instrument Company, Meriden, CT). Results are expressed as the mean stimulation index (SI), which is defined as the counts per minute $(\mathrm{cpm})$ of stimulated cells divided by the cpm of unstimulated cells. SI values above 2.0 were considered to be relevant.

The phenotype of proliferating cells was determined as described previously (Kap et al. 2008). Briefly, $4 \times 10^{6}$ viable MNC from ALN or spleen were suspended in $1 \mathrm{ml}$ PBS and incubated for $7 \mathrm{~min}$ at room temperature with CFSE (final concentration 1.5 $\mu \mathrm{M}$; Fluka, Deisenhofen, Germany). Labeled MNC were cultured for 7 days with rhMOG or MOG peptides. For flow cytometric analysis we used the following commercially available labeled monoclonal antibodies directed against human CD markers: Alexa Fluor 700-labeled anti-CD3 (BD Biosciences), APC-labeled anti-CD4 (DAKO, Glostrup, Denmark), biotinylated anti-CD8 (Serotec, Düsseldorf, Germany), and streptavidin PerCP (BD Biosciences). Flow cytometric analysis was performed on a FACSort using FACSDiva software (BD Biosciences). 
Statistics

Log-rank test, one-way ANOVA, and student's $t$ test were performed to statistically analyze the results. Results were considered statistically different at $p<0.05$.

\section{Results}

Plasma levels of anti-IL-17A mAb

in rhMOG-immunized monkeys

The marmosets included in the study were randomly assigned to three treatment groups. The control group $(n=8)$ received $1 \mathrm{ml} / \mathrm{kg}$ PBS and the two treatment groups received anti-IL-17A mAb at $3 \mathrm{mg} / \mathrm{ml} / \mathrm{kg}(n=8)$ or $30 \mathrm{mg} /$ $\mathrm{ml} / \mathrm{kg}(n=7)$. The test substances were subcutaneously administered once per week starting 1 day before immunization. Plasma levels of the anti-IL-17A mAb were determined 7 days after each administration (Fig. 1). As expected, plasma concentrations of the antibody in the $3 \mathrm{mg} / \mathrm{kg}$ antibody dose group were about tenfold lower compared with the $30 \mathrm{mg} / \mathrm{kg}$ antibody dose group. In two animals (M04099 and M03144) of the $3 \mathrm{mg} / \mathrm{kg}$ antibody dose group plasma antibody trough levels, measured 1 week after administration, were remarkably low, i.e., below $1 \mu \mathrm{g} / \mathrm{ml}$ at psd 34 , while these were above $1 \mu \mathrm{g} / \mathrm{ml}$ in all the other monkeys of this group. In the $30 \mathrm{mg} / \mathrm{kg}$ antibody dose animals, the plasma levels of the test substance were above $10 \mu \mathrm{g} / \mathrm{ml}$ from psd 6 throughout the experiment.

\section{Clinical signs after anti-IL-17A mAb treatment}

EAE was induced by a single immunization with rhMOG emulsified in CFA. The course of clinical signs in individual animals is depicted in Fig. 2. Solid lines

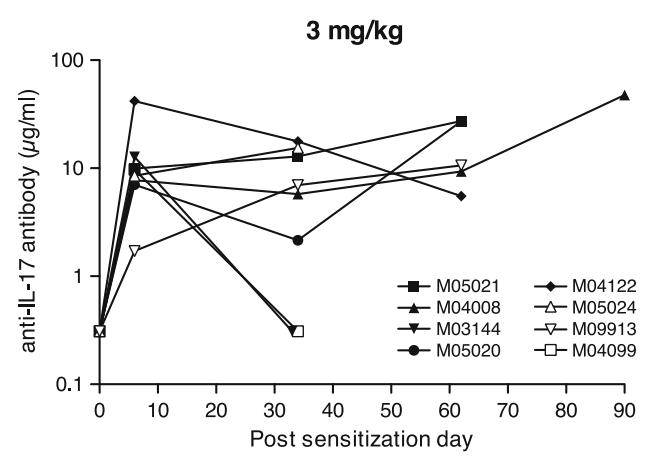

Fig. 1 Plasma levels of anti-human IL-17A mAb were sustained during the study. Once a week, animals received a subcutaneous injection with $1 \mathrm{ml} / \mathrm{kg}$ PBS (not shown), $3 \mathrm{mg} / \mathrm{kg}$ anti-IL-17A mAb (left graph), or $30 \mathrm{mg} / \mathrm{kg}$ anti-IL-17A mAb (right graph) starting 1 day before immunization. Plasma was collected from venous blood at several time points during the study and anti-IL-17A mAb levels in represent clinical scores and dotted lines represent percentage body weight loss, serving as a surrogate disease marker in this model. The figure shows a similar disease course in the monkeys that developed full-blown clinical EAE. Three monkeys from the $3 \mathrm{mg} / \mathrm{kg}$ antibody dose group and one monkey in the $30 \mathrm{mg} / \mathrm{kg}$ antibody dose group did not reach the stage of hind-limb paralysis (score 3) within the predetermined observation period of 113 days psd. In the $3 \mathrm{mg} / \mathrm{kg}$ antibody dose group one monkey (M05020) did not develop any signs of EAE within the pre-defined observation period. A second monkey of this group (M9913) reached EAE score 2 around psd 85, remitted and developed a second episode of score 2 EAE which remained present until the end of the observation period (psd 106). Two monkeys (M04008 and M05004) had to be sacrificed for ethical reasons with score 2 EAE due to serious body weight loss. It is noteworthy that the two monkeys with the lowest anti-IL-17A antibody plasma levels at day 34 (M03144 and M04099) were amongst the first monkeys to be sacrificed.

The EAE progression rate to overt neurological deficit was defined as the time interval between the day of rhMOG/CFA inoculation (psd 0) and the first day that clinical score 2 was observed. At the level of this parameter we observed a delayed EAE progression in the $3 \mathrm{mg} / \mathrm{kg}$ antibody dose group (mean psd, 69.3; range, 48-113) compared with the placebo group (mean psd, 57.1; range, 39-91) and the $30 \mathrm{mg} / \mathrm{kg}$ antibody dose group (mean psd, 60.0 ; range, 51-69). It is noteworthy that the variation between animals of this parameter was much lower in the $30 \mathrm{mg} / \mathrm{kg}$ antibody dose group (18 days) than in the placebo group (52 days) and the $3 \mathrm{mg} / \mathrm{kg}$ antibody dose group ( $>65$ days).

The disease progression from EAE score 2 (ataxia) to the ethical end-points at score 3.0 (paraplegia) or excessive body weight loss $(>20 \%)$ was more rapid in placebo group

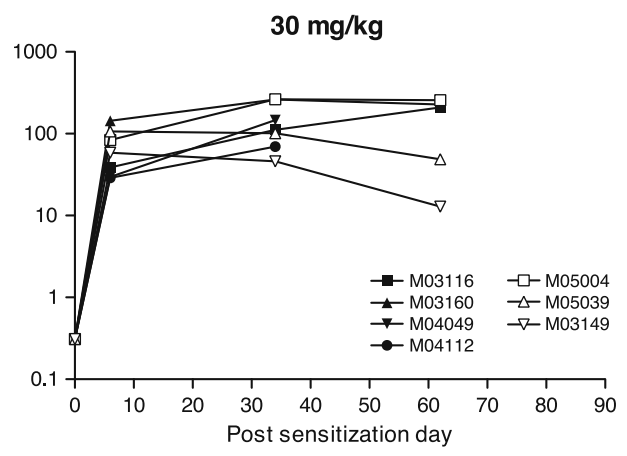

plasma were determined by ELISA. The $y$-axis have log scales. As expected, the anti-IL-17A mAb plasma levels in the control animals were throughout the experiment similar to the value on postsensitization day 0 of the treated animals (not shown). M04099 and M03144 of the low antibody dose group were not able to sustain a trough level of the antibody above $1 \mu \mathrm{g} / \mathrm{ml}$ 


\section{Control}
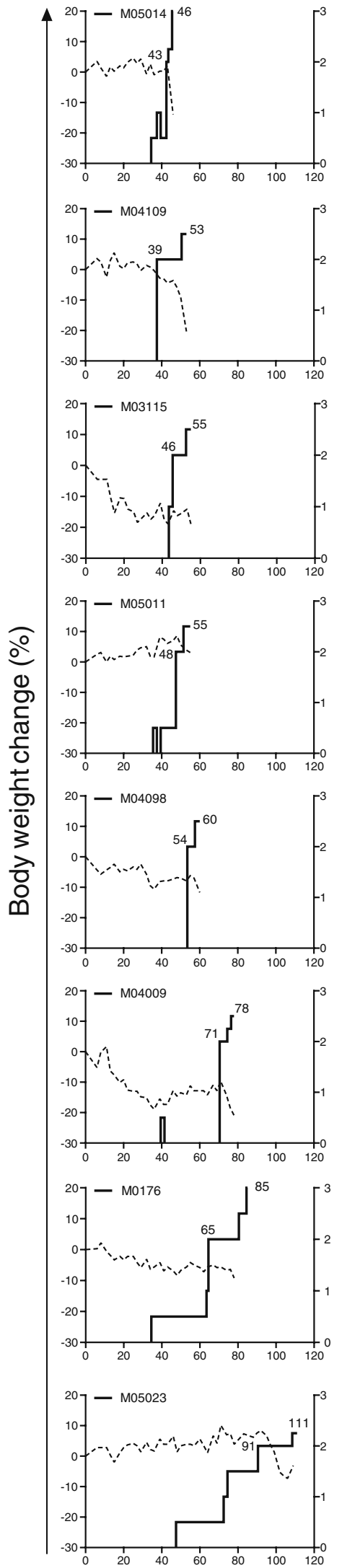

$3 \mathrm{mg} / \mathrm{kg}$

\section{$30 \mathrm{mg} / \mathrm{kg}$}
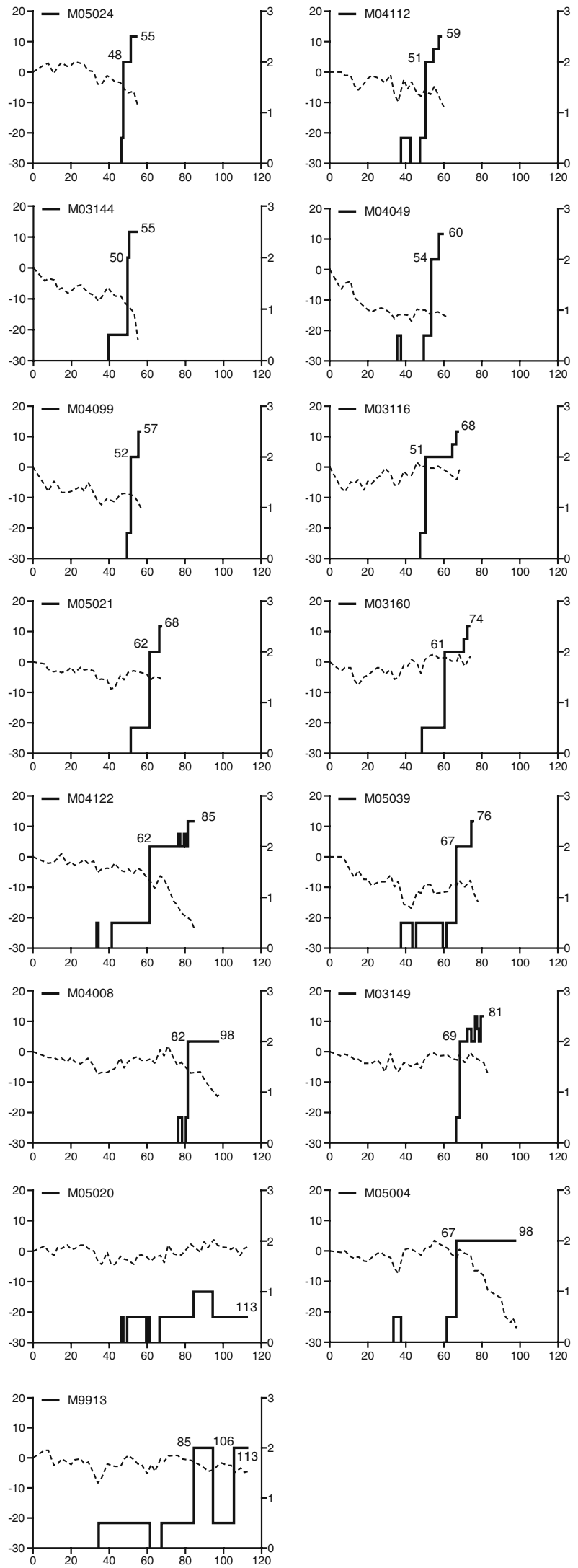
4Fig. 2 Clinical score and body weight loss of rhMOG-immunized marmosets. Shown are the clinical score (solid line, right $y$-axis) and the body weight change in percentages compared with day 0 (dotted line, left $y$-axis) of the placebo group (left graphs), the low dose group (middle graphs), and the high-dose group (right graphs). Numbers in the figure represent the time points (psd) when neurological signs (score $\geq 2$ ) were first observed and the day of sacrifice

monkeys (mean, 10.8 days; range, 3-20) than in monkeys from the $3 \mathrm{mg} / \mathrm{kg}$ antibody (mean, 12.9; range, 5-28) and $30 \mathrm{mg} / \mathrm{kg}$ antibody (mean, 13.7; range, 6-31) dose group. Statistical evaluation of both disease phases (score, $0-2$ and score, 2 to $>2$ ) was done by survival analysis, but no significant differences were found (Fig. 3).

In summary, these data show a moderate inhibitory effect of the antibody treatment on the EAE course, although differences between the groups did not reach statistical significance.

Effect of anti-IL-17A mAb on white matter lesion load

Prior to sectioning for histological examination, postmortem T2-weighted MR images were made of the formalin-fixed cerebral hemispheres to visualize and quantitate the total lesion load. The analyzed parameters were lesion volume, $\mathrm{T}_{2}$, and MTR values of white matter lesions. In fixed brains, an increased $\mathrm{T}_{2}$ signal reflects demyelination, while a reduction of MTR values correlates well with the intensity of macrophage infiltration (Blezer et al. 2007).

Significant differences were not observed between the three groups (Table 1). In the analyzed hemispheres of two animals of the control group cerebral white matter lesions were absent. White matter lesion volumes were slightly increased in the $30 \mathrm{mg} / \mathrm{kg}$ antibody dose group compared with the placebo group. Especially monkey M05004 had clinical score 2 for a long time, which might explain the high lesion load. The lesions in the 3 and $30 \mathrm{mg} / \mathrm{kg}$

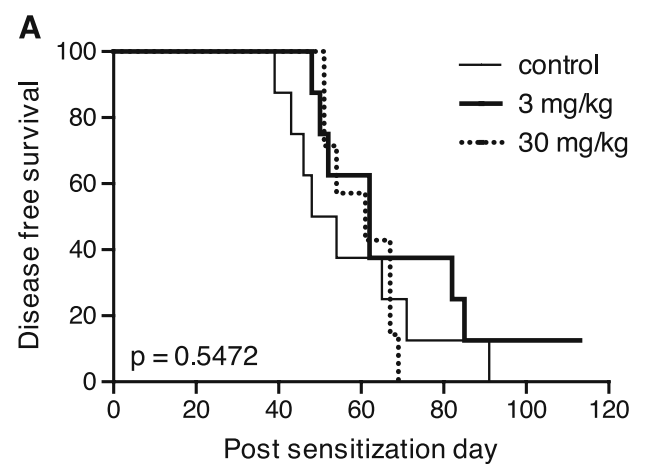

Fig. 3 Survival curves. Survival time to score 2 (a) and survival time to day of sacrifice (b) are shown. $p$ Values indicated in the graph are the results of comparing three groups. Comparing the survival to the day of sacrifice of the control group versus only the $3 \mathrm{mg} / \mathrm{kg}$ group resulted in a $p$ value of 0.1610 . When two animals of the $3 \mathrm{mg} / \mathrm{kg}$ antibody dose group had a slightly higher $\mathrm{T}_{2}$ and lower MTR value compared with placebo (Table 1).

These results were confirmed by the quantitative immunohistochemistry data (Table 1). Both inflammation and demyelination were moderately increased, although not significant, in the two antibody-treated groups compared with the placebo group.

For the correct interpretation of these data it is pertinent to emphasize that lesion formation in this model starts well before neurological deficits can be observed. Moreover, most animals were killed at the stage of clinically evident EAE, which occurred at different time intervals after immunization. It may therefore not be surprising that major effects of the antibody treatment on the CNS pathology were not observed, neither in positive nor in negative direction.

\section{IL-17A expression in the brain and lymphoid organs}

To assess the effect of anti-IL-17A mAb treatment on in situ expression of IL-17A, cryosectioned brains and lymphoid organs were examined with immunohistochemistry. In the brains of control and anti-IL-17A mAb-treated animals IL-17A-positive cells were found in the perivascular space or in the vicinity of blood vessels (Fig. 4a). No differences were observed between placebo and anti-IL-17A mAb-treated animals.

IL-17A-producing cells in secondary lymphoid organs were mainly found in the red pulp of the spleen and in the medulla of the lymph nodes. We observed a trend towards higher numbers of IL-17A producing cells in the spleen of animals dosed with $3 \mathrm{mg} / \mathrm{kg}$ anti-IL-17A mAb compared with animals that had received placebo or $30 \mathrm{mg} / \mathrm{kg}$ antibody, but differences were not significant (Fig. 4a-b). By contrast, we detected lower numbers of IL-17A producing cells in the ALN and ILN, which drain the immunization sites, and the brain-draining CLN, in both treatment groups compared with the placebo group (Fig. $4 a-b$ ). Taken together, these

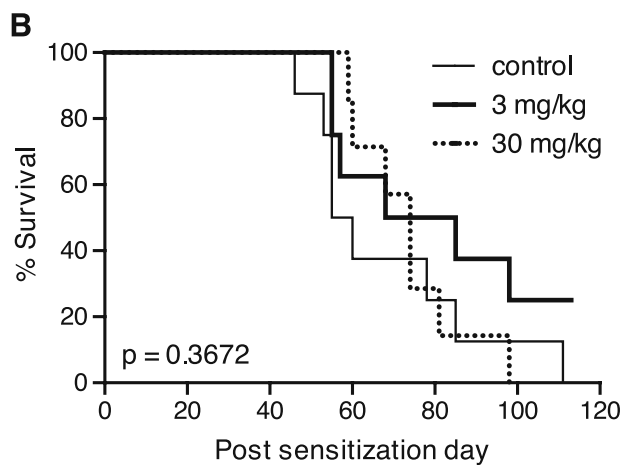

group with low anti-IL-17A mAb plasma levels (M04099 and M03144) are included in the control group instead of the $3 \mathrm{mg} / \mathrm{kg}$ group, the $p$ value is 0.0428 . According to the Bonferroni correction this is not significant, but it is highly suggestive for a delay in disease progression 
Table 1 White matter lesion parameters

\begin{tabular}{|c|c|c|c|c|c|c|}
\hline \multirow[t]{2}{*}{ Animal } & \multicolumn{3}{|c|}{ WM lesions assessed by MRI } & \multicolumn{3}{|l|}{ Histology } \\
\hline & Volume (mm3) & $\mathrm{T} 2$ (ms) & MTR & \# Inflammatory lesions/cm2 & \# Demyelinating lesions/cm2 & Demyelination \% \\
\hline \multicolumn{7}{|l|}{ Control } \\
\hline M0176 & 87 & 28.0 & 31.3 & 16 & 4.7 & 1.5 \\
\hline M03115 & 597 & 24.0 & 32.0 & 20 & 9.2 & 1.5 \\
\hline M04009 & 150 & 29.7 & 30.3 & 15 & 3.7 & 0.8 \\
\hline M04098 & 0 & - & - & 1 & 0.2 & 0 \\
\hline M04109 & 163 & 26.0 & 32.1 & 19 & 5.3 & 1.6 \\
\hline M05011 & 2 & 23.2 & 35.9 & 3 & 1.0 & 0.1 \\
\hline M05014 & 0 & - & - & 0 & 0.0 & 0 \\
\hline M05023 & 449 & 27.9 & 31.2 & 60 & 6.2 & 1.3 \\
\hline Mean & 181 & 26.5 & 32.1 & 17 & 3.8 & 0.9 \\
\hline SEM & 79 & 1.0 & 0.8 & 7.9 & 1.3 & 0.3 \\
\hline \multicolumn{7}{|l|}{$3 \mathrm{mg} / \mathrm{kg}$} \\
\hline M03144 & 483 & 29.9 & 29.9 & 32 & 7.7 & 1.6 \\
\hline M04008 & 135 & 27.6 & 29.2 & 25 & 9.8 & 1.6 \\
\hline M04099 & 113 & 26.6 & 33.9 & 11 & 1.7 & 0.3 \\
\hline M04122 & 354 & 27.6 & 31.2 & 45 & 12.0 & 2.4 \\
\hline M05020 & 178 & 26.1 & 33.3 & 19 & 3.3 & 0.4 \\
\hline M05021 & 30 & 29.3 & 30.9 & 5 & 2.0 & 0.2 \\
\hline M05024 & 642 & 27.5 & 31.2 & 52 & 11.0 & 2 \\
\hline M9913 & 321 & 25.9 & 29.7 & 13 & 4.3 & 3.3 \\
\hline Mean & 282 & 27.5 & 31.2 & 25 & 6.5 & 1.5 \\
\hline SEM & 73 & 0.5 & 0.6 & 5.9 & 1.5 & 0.4 \\
\hline \multicolumn{7}{|l|}{$30 \mathrm{mg} / \mathrm{kg}$} \\
\hline M03116 & 39 & 27.0 & 32.8 & 6 & 2.5 & 0.3 \\
\hline M03149 & 304 & 31.9 & 28.7 & 5 & 1.0 & 0.2 \\
\hline M03160 & 50 & 32.8 & 28.6 & 45 & 8.7 & 1.1 \\
\hline M04049 & 212 & 25.9 & 32.3 & 68 & 4.7 & 1.1 \\
\hline M04112 & 354 & 27.6 & 31.2 & 34 & 8.7 & 3 \\
\hline M05004 & 1261 & 25.8 & 29.7 & 52 & 8.8 & 3.4 \\
\hline M05039 & 715 & 27.1 & 31.5 & 18 & 3.2 & 0.7 \\
\hline Mean & 419 & 28.3 & 30.7 & 33 & 5.4 & 1.4 \\
\hline SEM & 165 & 1.1 & 0.6 & 9.1 & 1.3 & 0.5 \\
\hline
\end{tabular}

data suggest that the antibody treatment caused altered distribution of IL-17A producing cells over the different lymphoid compartments.

Effect of anti-IL-17A mAb on $\mathrm{T}$ cell proliferation

Cellular immune parameters of the three groups were examined to underpin the observed in vivo effects of the IL-17A mAb. To this end, PBMC and MNC of spleen and ALN were tested for proliferation against rhMOG and a panel of 23-mer overlapping MOG peptides.

Similar to previous studies (Kap et al. 2008), proliferation of PBMC during the disease course and at necropsy was low and remained confined to the immunizing rhMOG protein and a few peptides, i.e. $\mathrm{MOG}_{14-36}, \mathrm{MOG}_{24-46}$, and $\mathrm{MOG}_{34-56}$ (Fig. 5). No clear differences were observed between the three groups.

More robust proliferative responses were observed in the lymphoid organs, especially in the ALN and spleen. Figure 5 shows that in most animals the highest proliferative response was against rhMOG followed by peptides $\mathrm{MOG}_{14-36}$ and $\mathrm{MOG}_{24-46}$. MNC from the ALN of the anti-IL-17A mAbtreated groups displayed a lower proliferative response against rhMOG and MOG peptides than MNC from ALN of the placebo group (Fig. 5). No differences between the three groups were observed in the spleen (Fig. 5).

Next, we analyzed the phenotype of the rhMOG responsive cells using the CFSE fluorescent dye dilution 
Fig. 4 Differential expression of IL-17A in the brain and lymphoid organs. IL-17A expression was detected by immunohistochemistry. a $(200 \times)$ Representative examples of each group for brain, spleen, and axillary $(A L N)$, inguinal $(I L N)$, and cervical $(C L N)$ lymph nodes. b IL-17A positive cells per $\mathrm{mm}^{2}$ (mean \pm SEM). Only one CLN of the control group was analyzed. No IL-17A producing cells were detected in the ILN of the $30 \mathrm{mg} / \mathrm{kg}$ treated group. Statistical differences between the numbers of IL-17A positive cells were analyzed by One-way ANOVA. Results were considered statistically different at $p<0.05$. No statistical differences were found between the three groups
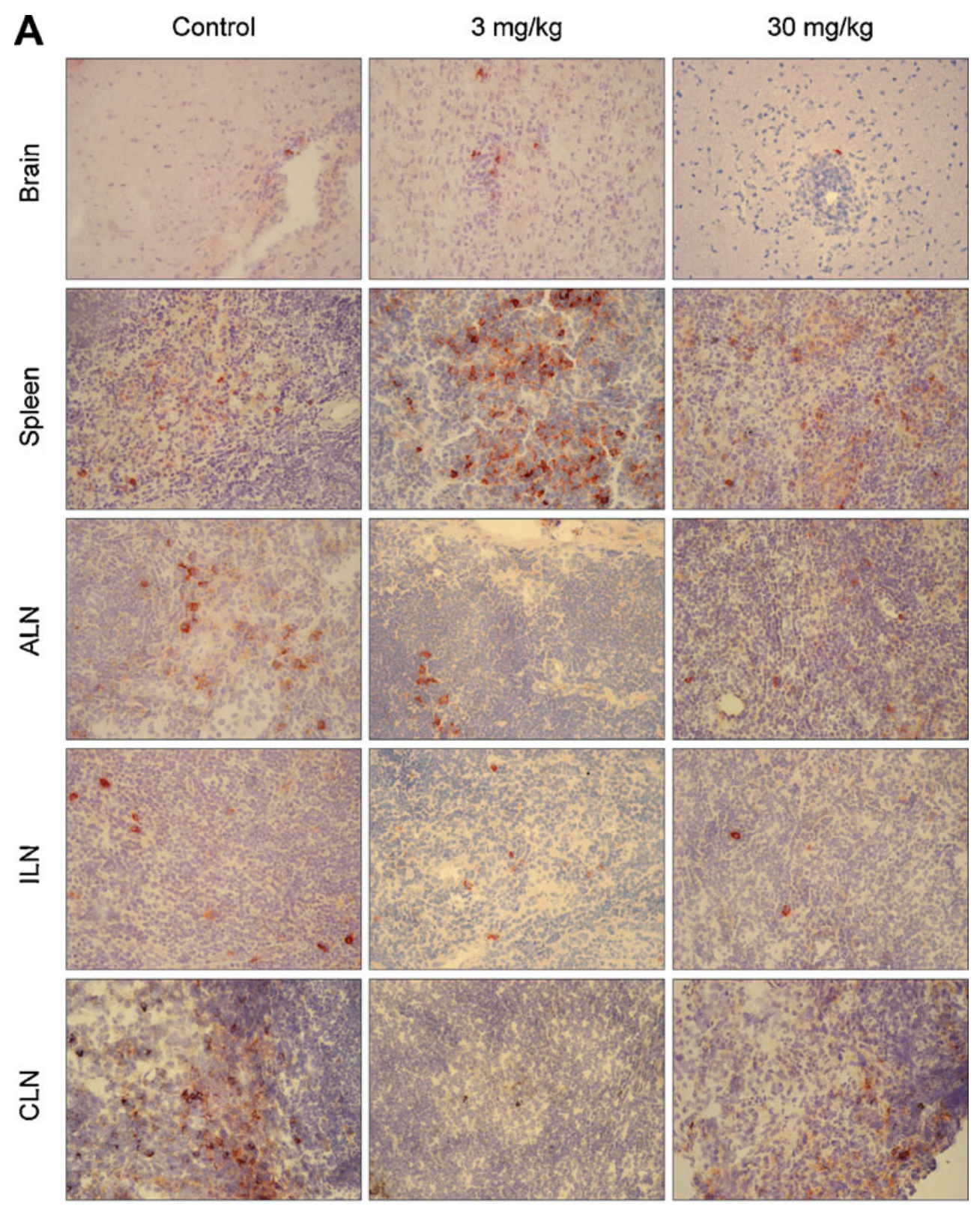

B

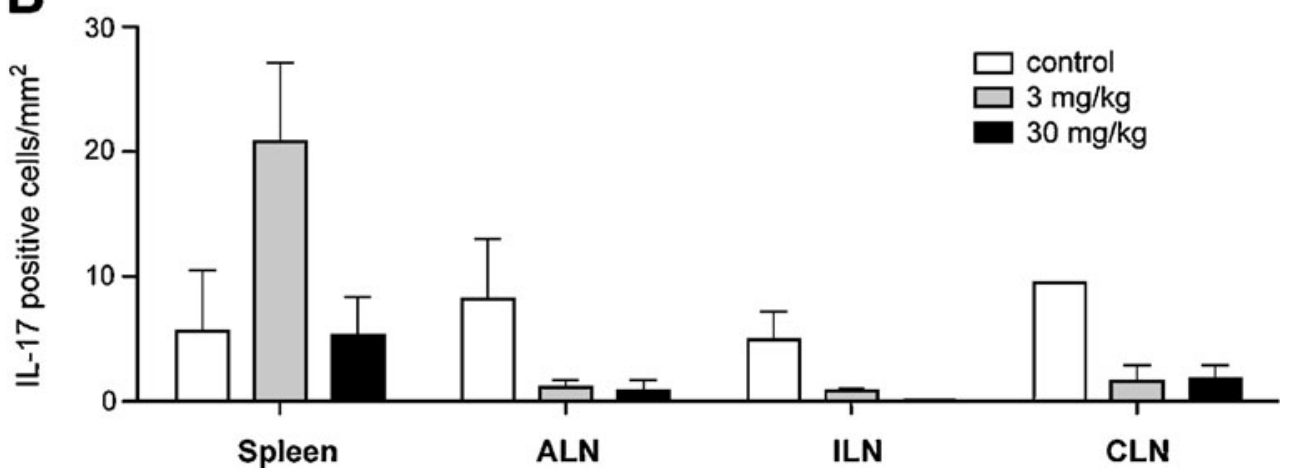




\begin{tabular}{|c|c|c|c|c|c|c|c|c|c|c|c|c|c|}
\hline & & \multicolumn{4}{|c|}{ PBMC } & \multicolumn{4}{|c|}{ Spleen } & \multicolumn{4}{|c|}{ ALN } \\
\hline & & \multirow[b]{2}{*}{ rhMOG } & \multicolumn{3}{|c|}{ MOG peptide } & \multirow[b]{2}{*}{ rhMOG } & \multicolumn{3}{|c|}{ MOG peptide } & \multirow[b]{2}{*}{ rhMOG } & \multicolumn{3}{|c|}{ MOG peptide } \\
\hline & & & $14-36$ & $24-46$ & $34-56$ & & $14-36$ & $24-46$ & $34-56$ & & $14-36$ & $24-46$ & $34-56$ \\
\hline \multirow{8}{*}{ Control } & M0176 & & & & & & & & & & & & \\
\hline & M03115 & & & & & & & & & & & & \\
\hline & M04009 & & & & & & & & & & & & \\
\hline & M04098 & & & & & & & & & & & & \\
\hline & M04109 & & & & & & & & & & & & \\
\hline & M05011 & & & & & & & & & & & & \\
\hline & M05014 & & & & & & & & & & & & \\
\hline & M05023 & & & & & & & & & & & & \\
\hline \multirow{8}{*}{$3 \mathrm{mg} / \mathrm{kg}$} & M03144 & & & & & & & & & & & & \\
\hline & M04008 & & & & & & & & & & & & \\
\hline & M04099 & & & & & & & & & & & & \\
\hline & M04122 & & & & & & & & & & & & \\
\hline & M05020 & & & & & & & & & & & & \\
\hline & M05021 & & & & & & & & & & & & \\
\hline & M05024 & & & & & & & & & & & & \\
\hline & M9913 & & & & & & & & & & & & \\
\hline \multirow{7}{*}{$30 \mathrm{mg} / \mathrm{kg}$} & M03116 & & & & & & & & & & & & \\
\hline & \begin{tabular}{|l|} 
M03149 \\
\end{tabular} & & & & & & & & & & & & \\
\hline & M03160 & & & & & & & & & & & & \\
\hline & M04049 & & & & & & & & & & & & \\
\hline & M04112 & & & & & & & & & & & & \\
\hline & M05004 & & & & & & & & & & & & \\
\hline & M05039 & & & & & & & & & & & & \\
\hline
\end{tabular}

\begin{tabular}{|c|c|}
\hline $\mathrm{S} \mid<2$ & \\
\hline $2 \leq \mathrm{S} \mid<4$ & \\
\hline $4 \leq \mathrm{S} \mid<6$ & \\
\hline $\mathrm{SI} \geq 6$ & \\
\hline
\end{tabular}

Fig. $5 \mathrm{~T}$ cell responses after anti-IL-17A mAb treatment. PBMC and mononuclear cells isolated from the axillary lymph node and spleen at necropsy were tested for proliferation against rhMOG and MOG peptides. The stimulation index (SI) was calculated by dividing the

technique in combination with cell phenotyping for surface markers. RhMOG responsive $\mathrm{CD} 3^{+} \mathrm{CD} 4^{+}$and $\mathrm{CD} 3^{+} \mathrm{CD} 8^{+}$ cells with CFSE dye dilution were visualized by flow cytometry. The percentages of $\mathrm{CD}^{+} \mathrm{CD}^{+}$and $\mathrm{CD}^{+} \mathrm{CD} 8^{+}$ cells determined by fluorescent dye dilution did not differ significantly between placebo and anti-IL-17A mAb-treated groups (data not shown), suggesting that the treatment with anti-IL-17A mAb did not cause a skewing of the CD4/CD8 ratio of rhMOG-specific $\mathrm{T}$ cells.

In conclusion, treatment with the anti-IL-17A antibody did not exert an obvious effect on the quality or quantity of the cellular autoimmune reactions. However, we like to emphasize again that the analyzed lymphoid organs were collected at different time intervals after the EAE induction, creating a possible bias in the data.

\section{Discussion}

Key pathogenic elements for EAE development in rhMOGimmunized marmosets comprise CD40, IL-12, and IL-23 ('t Hart et al. 2005a; Boon et al. 2001; Brok et al. 2002; Laman et al. 2002). We have shown that an antibody counts per minute (cpm) of stimulated wells by cpm of unstimulated wells. The stimulation index is divided into groups with different shading as indicated in the figure

against the shared p40 subunit of IL-12 and IL-23 caused profound suppression of disease symptoms when dosed from disease induction. One possible mechanism of action could have been a reduction in IL-17A that is one of the downstream cytokines from IL-23. However, the modest effect of an anti-IL-17A antibody in the current study indicates that a reduction in IL-17A does not account for the activity of IL-12 p40 antibody and that other cytokines, perhaps IL-12 directly or other cytokines downstream of IL-12 and/or IL-23, are more important in the induction phase of this non-relapsing model.

The results of the current study demonstrate that prophylactic treatment with an anti-IL-17A mAb induced only a moderate extension of the disease-free time interval after EAE induction. Furthermore, immune parameters of the disease, such as $\mathrm{T}$ cell proliferation against rhMOG or MOG peptides was only somewhat reduced, whereas also a marginal aggravation of CNS pathology was observed. However, the variations were all within the normal range of variation in this outbred model and may also be attributable to the longer survival. Using immunohistochemistry only a few IL-17A-positive cells could be detected in the brains of placebo and treated monkeys, but no significant differences 
between groups were observed. The data show a trend towards increased numbers of IL-17A-positive cells in the spleen in the $3 \mathrm{mg} / \mathrm{kg}$ group and a decreased number in the axillary and inguinal lymph nodes of both groups compared with placebo animals (not significant). The higher number of Th17 cells in the spleen of monkeys that have been treated with anti-IL-17A antibody compared with placebo monkeys may be explained by feedback regulation. The observation that IL-17A receptor deficient mice produce more IL-17A (Smith et al. 2008) indicates that IL-17A production may be regulated by a negative feedback loop. In conclusion, the current results fail to show a robust effect of the anti-IL-17A mAb treatment on the EAE course in rhMOG/CFA-immunized marmosets.

The moderate protection against EAE induction is in agreement with data reported by some groups in mouse EAE models. Also in IL-17A $\mathrm{A}^{-/}$mice (Komiyama et al. 2006), in mice treated with anti-IL-17A mAb (Chen et al. 2006; Hofstetter et al. 2005; Langrish et al. 2005), and in mice vaccinated against IL-17A (Rohn et al. 2006) only moderately delayed onset of clinical signs has been observed. However, other groups have demonstrated more robust effects of IL-17A on the EAE course in mice. Examples are by vaccination with IL-17A-OVA complexes that induce IL-17A blocking antibodies (Uyttenhove and Van Snick 2006) or adoptive transfer of IL-17 $7^{+}$and IL-17 $\mathrm{T}$ cells (Jager et al. 2009; Komiyama et al. 2006; Langrish et al. 2005). Furthermore, in the chronic-relapsing EAE model in Biozzi ABH mice, prophylactic dosing with an anti-IL-17A antibody produced a small delay in onset of the acute phase of disease, whereas treatment with the antibody started after the acute phase or during the secondaryprogressive phase was very efficacious, and could even reverse the chronic motor disability (Smith, unpublished observations). Other groups suggested that Th17 are not pathogenic at all (Haak et al. 2009) or that besides Th17 cells, Th1 cells are also essential in EAE (Kroenke et al. 2008; Lees et al. 2008; O'Connor et al. 2008).

How can it be explained that IL-17A neutralization does not fully protect against EAE in the rhMOG-induced marmoset model? One possible explanation is the high complexity of the model. Unraveling of the pathogenic mechanism showed that the disease is initiated by $\mathrm{MOG}_{24}$

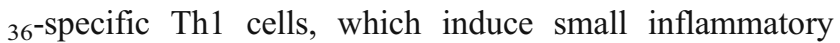
lesions (Brok et al. 2000). The moderate inflammatory injury caused by Th1 cells can be dramatically amplified by antibodies against conformational epitopes of MOG (Genain et al. 1995), which are clearly produced in the rhMOG model. Another subset of highly reactive $\mathrm{T}$ cells with natural killer-cytotoxic T-lymphocyte characteristics and specific for $\mathrm{MOG}_{34-56}$ becomes involved at a later stage in the disease (Kap et al. 2008). Our previously reported studies in marmoset EAE models induced with $\mathrm{MOG}_{34-56}$ in CFA (Kap et al. 2008) or IFA (Jagessar et al. 2010) demonstrated that the same clinical and pathological endpoints can be reached via different immunopathogenic routes. It can thus be envisaged that after neutralization of IL-17A in the complex rhMOG/CFA-induced model other pathogenic mechanisms, such as Th1 cells, may come into play. It could also be possible that Th1 and Th17 cells act in different phases of the disease. In the previously reported study in the rhMOG marmoset model it was observed that Th1 cells specific for $\mathrm{MOG}_{24-36}$ are engaged in EAE

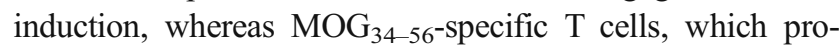
duce mainly IL-17A, mediate disease progression (Jagessar et al. 2010; Kap et al. 2008). This suggests that, consistent with the results in the Biozzi ABH mouse model, neutralizing IL-17A in a later phase of the disease is more effective.

Yet, another possible explanation is that IL-17A, although being the signature cytokine of Th17 cells, is not the dominant pathogenic factor produced by Th17 cells. This explanation is supported by data in mice. IL-12/IL$23 \mathrm{p} 40^{-/-}$mice are completely resistant to EAE, whereas only clinical scores and not the disease incidence is reduced in IL-17 $7^{-1-}$ mice (Becher et al. 2002; Cua et al. 2003; Gran et al. 2002; Komiyama et al. 2006). Moreover, treatment of mice with anti-IL-23 antibody prevented either EAE induction or relapse, in contrast to the anti-IL-17A antibody treatment that blocked relapse, but had a smaller effect on the induction phase (Chen et al. 2006). This may suggest that other Th17 cytokines induced by IL-23 may be pathogenetically more relevant than IL-17A in the induction phase, such as IL-21 or IL-22 (reviewed in (Fouser et al. 2008)). In addition, Chen et al. suggested that IL-23 may also directly activate macrophages to produce inflammatory cytokines, such as IL-6, IL-1, and TNF- $\alpha$, which may cause inflammation and demyelination even when IL-17A is neutralized (Chen et al. 2006).

Is there a place for an IL-17A neutralizing antibody in the treatment of MS? Axtell et al. reported that on the basis of clinical response to IFN- $\beta$ two groups may be distinguished in the MS patient population. Responsiveness to IFN- $\beta$ treatment requires a Th1 cytokine profile, whereas cases with a Th17 dominated cytokine profile are non-responders to IFN- $\beta$ treatment (Axtell et al. 2010). It is tempting to speculate on the basis of these and our data that non-responder cases to IFN- $\beta$ treatment in the MS population may benefit from treatment with IL17A antibody. The ideal study design to obtain proof-ofprinciple may be a parallel effectivity analysis in the marmoset EAE model, where in one sibling of a chimeric twin EAE is induced with $\mathrm{MOG}_{34-56}$ in CFA, which is a Th1 prone model (Jagessar et al. 2010; Kap et al. 2008) and in the other twin sibling with $\mathrm{MOG}_{34-56}$ in IFA, which is a more Th17 prone disease (Jagessar et al. 2010; Kap et al. 2008). 
In summary, we found that treatment with anti-IL-17A antibody induces a moderate delay of clinical EAE in marmosets, but that EAE is not completely abrogated. This suggests a pathogenic role for IL-17A in the marmoset EAE model and maybe in MS, but IL-17A may not be the only key pathogenic cytokine.

Acknowledgements The authors like to thank Fred Batenburg for excellent biotechnical assistance and daily care of the monkeys, Jaco Bakker DVM, Gerco Braskamp DVM and Merei Keehnen DVM for expert veterinary care, Tom Haaksma and Dr. Ivanela Kondova for autopsy of the monkeys. The authors thank Henk van Westbroek for the artwork. The anti-IL-17A antibody was provided by UCB Celltech (UK). The study was financially supported by UCB Celltech.

Conflicts of Interest The authors of this manuscript do not report conflict of interest.

Open Access This article is distributed under the terms of the Creative Commons Attribution Noncommercial License which permits any noncommercial use, distribution, and reproduction in any medium, provided the original author(s) and source are credited.

\section{References}

Axtell RC, de Jong BA, Boniface K, van der Voort LF, Bhat R, De Sarno P, Naves R, Han M, Zhong F, Castellanos JG, Mair R, Christakos A, Kolkowitz I, Katz L, Killestein J, Polman CH, de Waal MR, Steinman L, Raman C (2010) T helper type 1 and 17 cells determine efficacy of interferon-beta in multiple sclerosis and experimental encephalomyelitis. Nat Med 16:406-412

Becher B, Durell BG, Noelle RJ (2002) Experimental autoimmune encephalitis and inflammation in the absence of interleukin-12. J Clin Invest 110:493-497

Billiau A, Matthys P (2001) Modes of action of Freund's adjuvants in experimental models of autoimmune diseases. J Leukoc Biol 70:849-860

Blezer EL, Bauer J, Brok HP, Nicolay K, 't Hart BA (2007) Quantitative MRI-pathology correlations of brain white matter lesions developing in a non-human primate model of multiple sclerosis. NMR Biomed 20:90-103

Boon L, Brok HP, Bauer J, Ortiz-Buijsse A, Schellekens MM, RamdienMurli S, Blezer E, van Meurs M, Ceuppens J, de Boer M, 't Hart BA, Laman JD (2001) Prevention of experimental autoimmune encephalomyelitis in the common marmoset (Callithrix jacchus) using a chimeric antagonist monoclonal antibody against human CD40 is associated with altered B cell responses. J Immunol 167:2942-2949

Brok HP, Uccelli A, Kerlero De Rosbo N, Bontrop RE, Roccatagliata L, de Groot NG, Capello E, Laman JD, Nicolay K, Mancardi GL, Ben-Nun A, 't Hart BA (2000) Myelin/oligodendrocyte glycoprotein-induced autoimmune encephalomyelitis in common marmosets: the encephalitogenic T cell epitope pMOG24-36 is presented by a monomorphic MHC class II molecule. J Immunol 165:1093-1101

Brok HP, van Meurs M, Blezer E, Schantz A, Peritt D, Treacy G, Laman JD, Bauer J, 't Hart BA (2002) Prevention of experimental autoimmune encephalomyelitis in common marmosets using an anti-IL-12p40 monoclonal antibody. J Immunol 169:65546563
Chen Y, Langrish CL, McKenzie B, Joyce-Shaikh B, Stumhofer JS, McClanahan T, Blumenschein W, Churakovsa T, Low J, Presta L, Hunter CA, Kastelein RA, Cua DJ (2006) Anti-IL-23 therapy inhibits multiple inflammatory pathways and ameliorates autoimmune encephalomyelitis. J Clin Invest 116:1317-1326

Compston A, Coles A (2008) Multiple sclerosis. Lancet 372:15021517

Cua DJ, Sherlock J, Chen Y, Murphy CA, Joyce B, Seymour B, Lucian L, To W, Kwan S, Churakova T, Zurawski S, Wiekowski M, Lira SA, Gorman D, Kastelein RA, Sedgwick JD (2003) Interleukin-23 rather than interleukin-12 is the critical cytokine for autoimmune inflammation of the brain. Nature 421:744-748

Fouser LA, Wright JF, Dunussi-Joannopoulos K, Collins M (2008) Th17 cytokines and their emerging roles in inflammation and autoimmunity. Immunol Rev 226:87-102

Genain CP, Nguyen MH, Letvin NL, Pearl R, Davis RL, Adelman M, Lees MB, Linington C, Hauser SL (1995) Antibody facilitation of multiple sclerosis-like lesions in a nonhuman primate. J Clin Invest 96:2966-2974

Gran B, Zhang GX, Yu S, Li J, Chen XH, Ventura ES, Kamoun M, Rostami A (2002) IL-12p35-deficient mice are susceptible to experimental autoimmune encephalomyelitis: evidence for redundancy in the IL-12 system in the induction of central nervous system autoimmune demyelination. J Immunol 169:7104-7110

Haak S, Croxford AL, Kreymborg K, Heppner FL, Pouly S, Becher B, Waisman A (2009) IL-17A and IL-17F do not contribute vitally to autoimmune neuro-inflammation in mice. J Clin Invest 119:61-69

Hofstetter HH, Ibrahim SM, Koczan D, Kruse N, Weishaupt A, Toyka KV, Gold R (2005) Therapeutic efficacy of IL-17 neutralization in murine experimental autoimmune encephalomyelitis. Cell Immunol 237:123-130

Jager A, Dardalhon V, Sobel RA, Bettelli E, Kuchroo VK (2009) Th1, Th17, and Th9 effector cells induce experimental autoimmune encephalomyelitis with different pathological phenotypes. J Immunol 183:7169-7177

Jagessar SA, Kap YS, Heijmans N, van Driel N, van Straalen L, Bajramovic JJ, Brok HPM, Blezer ELA, Bauer J, Laman JD, Hart BA (2010) Induction of progressive demyelinating autoimmune encephalomyelitis in common marmoset monkeys using MOG3456 peptide in incomplete Freund adjuvant. J Neuropathol Exp Neurol 69:372-385

Kap YS, Smith P, Jagessar SA, Remarque E, Blezer E, Strijkers GJ, Laman JD, Hintzen RQ, Bauer J, Brok HP, 't Hart BA (2008) Fast progression of recombinant human myelin/oligodendrocyte glycoprotein (MOG)-induced experimental autoimmune encephalomyelitis in marmosets is associated with the activation of MOG34-56specific cytotoxic T cells. J Immunol 180:1326-1337

Kap YS, Laman JD, 't Hart BA (2010) Experimental Autoimmune Encephalomyelitis in the Common Marmoset, a Bridge Between Rodent EAE and Multiple Sclerosis for Immunotherapy Development. J Neuroimmune Pharmacol 5:220-230

Kerlero de Rosbo N, Hoffman M, Mendel I, Yust I, Kaye J, Bakimer R, Flechter S, Abramsky O, Milo R, Karni A, Ben-Nun A (1997) Predominance of the autoimmune response to myelin oligodendrocyte glycoprotein (MOG) in multiple sclerosis: reactivity to the extracellular domain of MOG is directed against three main regions. Eur J Immunol 27:3059-3069

Komiyama Y, Nakae S, Matsuki T, Nambu A, Ishigame H, Kakuta S, Sudo K, Iwakura Y (2006) IL-17 plays an important role in the development of experimental autoimmune encephalomyelitis. J Immunol 177:566-573

Kroenke MA, Carlson TJ, Andjelkovic AV, Segal BM (2008) IL12- and IL-23-modulated T cells induce distinct types of EAE based on histology, CNS chemokine profile, and response to cytokine inhibition. J Exp Med 205:1535-1541 
Laman JD, van Meurs M, Schellekens MM, de Boer M, Melchers B, Massacesi L, Lassmann H, Claassen E, 't Hart BA (1998) Expression of accessory molecules and cytokines in acute EAE in marmoset monkeys (Callithrix jacchus). J Neuroimmunol 86:30-45

Laman JD, 't Hart BA, Brok H, Meurs M, Schellekens MM, Kasran A, Boon L, Bauer J, Boer M, Ceuppens J (2002) Protection of marmoset monkeys against EAE by treatment with a murine antibody blocking CD40 (mu5D12). Eur J Immunol 32:2218-2228

Langrish CL, Chen Y, Blumenschein WM, Mattson J, Basham B, Sedgwick JD, McClanahan T, Kastelein RA, Cua DJ (2005) IL23 drives a pathogenic $\mathrm{T}$ cell population that induces autoimmune inflammation. J Exp Med 201:233-240

Lees JR, Iwakura Y, Russell JH (2008) Host T cells are the main producers of IL-17 within the central nervous system during initiation of experimental autoimmune encephalomyelitis induced by adoptive transfer of Th1 cell lines. J Immunol 180:8066-8072

O'Connor RA, Prendergast CT, Sabatos CA, Lau CW, Leech MD, Wraith DC, Anderton SM (2008) Cutting edge: Th1 cells facilitate the entry of Th17 cells to the central nervous system during experimental autoimmune encephalomyelitis. J Immunol 181:3750-3754

Rohn TA, Jennings GT, Hernandez M, Grest P, Beck M, Zou Y, Kopf M, Bachmann MF (2006) Vaccination against IL-17 suppresses autoimmune arthritis and encephalomyelitis. Eur J Immunol 36:2857-2867

Smith E, Stark MA, Zarbock A, Burcin TL, Bruce AC, Vaswani D, Foley P, Ley K (2008) IL-17A inhibits the expansion of IL-17Aproducing T cells in mice through "short-loop" inhibition via IL17 receptor. J Immunol 181:1357-1364

Sospedra M, Martin R (2005) Immunology of multiple sclerosis. Annu Rev Immunol 23:683-747 't Hart BA, Massacesi L (2009) Clinical, pathological, and immunologic aspects of the multiple sclerosis model in common marmosets (Callithrix jacchus). J Neuropathol Exp Neurol 68:341-355

't Hart BA, Bauer J, Muller HJ, Melchers B, Nicolay K, Brok H, Bontrop RE, Lassmann H, Massacesi L (1998) Histopathological characterization of magnetic resonance imaging-detectable brain white matter lesions in a primate model of multiple sclerosis: a correlative study in the experimental autoimmune encephalomyelitis model in common marmosets (Callithrix jacchus). Am J Pathol 153:649-663

't Hart BA, Laman JD, Bauer J, Blezer E, van Kooyk Y, Hintzen RQ (2004) Modelling of multiple sclerosis: lessons learned in a nonhuman primate. Lancet Neurol 3:588-597

't Hart BA, Blezer EL, Brok HP, Boon L, de Boer M, Bauer J, Laman JD (2005a) Treatment with chimeric anti-human CD40 antibody suppresses MRI-detectable inflammation and enlargement of preexisting brain lesions in common marmosets affected by MOGinduced EAE. J Neuroimmunol 163:31-39

't Hart BA, Brok HP, Remarque E, Benson J, Treacy G, Amor S, Hintzen RQ, Laman JD, Bauer J, Blezer EL (2005b) Suppression of ongoing disease in a nonhuman primate model of multiple sclerosis by a human-anti-human IL-12p40 antibody. J Immunol 175:4761-4768

't Hart BA, Hintzen RQ, Laman JD (2008) Preclinical assessment of therapeutic antibodies against human CD40 and human interleukin-12/23p40 in a nonhuman primate model of multiple sclerosis. Neurodegener Dis 5:38-52

Uyttenhove C, Van Snick J (2006) Development of an anti-IL-17A auto-vaccine that prevents experimental auto-immune encephalomyelitis. Eur J Immunol 36:2868-2874 